\title{
Ergothioneine, a metabolite of the gut bacterium Lactobacillus reuteri, protects against stress-induced sleep disturbances
}

\author{
Yoshiki Matsuda (1)', Nobuyuki Ozawa', Takiko Shinozaki ${ }^{1}$, Ken-ichi Wakabayashi', Kosuke Suzuki ${ }^{2}$, Yusuke Kawano (i) ${ }^{3}$,
} Iwao Ohtsu (10 ${ }^{4}$ and Yoshitaka Tatebayashi ${ }^{1}$

\begin{abstract}
The relationships between depression and gut microbiota, particularly those involving the immune system, have become a major focus of recent research. Here, we analyzed changes in gut microbiota and their sulfur metabolites in the feces of a depression rat model using the modified 14-day social defeat stress (SDS) paradigm. Our results showed that SDS increased fecal Lactobacillus reuteri in correlation with ergothioneine levels at around day 11, which continued for at least 1 month following SDS administration. In vitro study further revealed that L. reuteri is capable of producing ergothioneine. Although the known anti-inflammatory and anti-oxidative actions of ergothioneine suggested that the increased fecal ergothioneine levels may be related to intestinal anti-inflammatory defense mechanisms, no change was observed in the plasma ergothioneine levels during the same observation period, indicating that the defense mechanisms may not be sufficiently reflected in the body. As ergothioneine is a natural ingredient that is absorbed mainly from the upper gastrointestinal tract, we hypothesized that oral ergothioneine may exert antidepressant effects. As expected, oral administration of ergothioneine prior to and during the SDS paradigm had a preventative effect on SDS-induced depressive behaviors, such as social avoidance and depression-like sleep abnormalities, particularly those of rapid eye movement sleep. These findings indicate that ergothioneine, a metabolite of L. reuteri, may be a common substance in the microbiota-gut-brain axis that prevents stress-induced sleep disturbances, especially those associated with depression.
\end{abstract}

\section{Introduction}

Psychosocial stress is an environmental factor associated with the increased incidence of psychiatric illnesses such as major depressive disorder (MDD) ${ }^{1}$. In addition to the core symptoms (depressed mood and loss of interest/ pleasure), MDD can also be characterized by somatic symptoms such as sleep abnormalities ${ }^{2}$. We have recently developed a depression rat model using the 14-day social defeat stress (SDS) paradigm ${ }^{3}$. Compared to other rodent stress models, nearly all SDS rats exhibit long-term social

\footnotetext{
Correspondence: Yoshitaka Tatebayashi (tatebayashi-ys@igakuken.or.jp) ${ }^{1}$ Affective Disorders Research Project, Tokyo Metropolitan Institute of Medical Science, Tokyo, Japan

${ }^{2}$ Research Institute, Suntory Global Innovation Center Ltd., Kyoto, Japan Full list of author information is available at the end of the article
}

avoidance and MDD-like sleep abnormalities in our paradigm, which can be rescued by chronic antidepressant treatments ${ }^{3}$. In particular, the observed sleep abnormalities exhibit numerous similarities to those in patients with MDD. These include significantly increased rapid eye movement (REM) and decreased non-REM (NREM) sleep durations, increased sleep fragmentation, and decreased REM sleep latency during the light phase ${ }^{3}$.

Recent research has reported the influence of gut microbiota on cerebral function (i.e., the microbiota-gutbrain axis $)^{4-9}$. For example, depressive behaviors develop in germ-free rodents following fecal transplants from human patients with $\mathrm{MDD}^{10,11}$. On the other hand, decreases in MDD-like behaviors occurred following the administration of prebiotics in mice subjected to mild 
chronic stress ${ }^{12}$. More recently, Pearson-Leary et al. demonstrated that the gut microbiota regulate the increases in MDD-like behaviors and inflammatory processes in the ventral hippocampus of SDS-vulnerable rats $^{13}$. Nevertheless, considerably less research is available regarding stress-induced changes in gut bacteriaproduced metabolites, especially sulfur metabolites, or their effects on cerebral functions.

Despite its essential involvement in all organisms, our understanding of sulfur metabolism lags behind that of carbon or nitrogen, mostly due to difficulty in metabolite detection. Cellular sulfur metabolites are quantitatively scarce and readily undergo redox reactions on their thiol group. Recently, however, the combination of sensitive liquid chromatography-tandem mass spectrometry (LCMS/MS) with thiol-specific derivatization methods using monobromobimane has enabled such detection ${ }^{14-17}$. Here, we analyzed how SDS influences gut microbiota and their sulfur metabolites in rats and found that fecal Lactobacillus reuteri increased concomitantly with ergothioneine during the late SDS stage. To evaluate the role of ergothioneine, we preventatively administered ergothioneine to the SDS rats prior to and during SDS and analyzed its effects on the depressive behaviors and sleep abnormalities in our SDS rat model.

\section{Materials and methods \\ Animals}

All procedures were approved by the Animal Use and Care Committee of the Tokyo Metropolitan Institute of Medical Science for Ethics of Animal Experimentation. Animals were kept under standard laboratory conditions [12 h light/dark cycle, lights on at 08:00 (= Zeitgeber time 0 ; ZT0)] with food and water available ad libitum unless otherwise indicated. All animal experiments were performed in 2015-2018.

\section{Social defeat paradigm}

We applied a modified repeated SDS model ${ }^{3}$ for 14 consecutive days using male Sprague Dawley (SD) rats that were $\sim 8$ weeks old (Charles River Laboratories Japan, Yokohama, Japan) at stress onset. Briefly, each SD rat was transferred into the home cage of a retired aggressive male Brown Norway (BN) rat (>7 months of age; Charles River Laboratories Japan). The resident $\mathrm{BN}$ rat was allowed direct physical contact with the SD rat (intruder) for $10 \mathrm{~min}$, and then resident and intruder rats were kept in indirect contact for $24 \mathrm{~h}$ in a resident cage using a perforated clear divider to prevent physical contact. The next day, the intruder was exposed to a novel resident BN aggressor. Subsequently, intruders were subjected to combined stress (direct and indirect contact) for the first 5 weekdays, followed by only indirect contact for the subsequent 2 weekend days. This process was continuously repeated for 2 weeks. Control animals were housed on one side of a perforated divider without a resident. Upon termination of SDS, all rats were housed individually.

\section{Social interaction}

The social interaction test was conducted to assess social avoidance behavior ${ }^{3,18,19}$. Briefly, the arena was an open field $(90 \times 90 \times 45 \mathrm{~cm})$ maintained in weak lighting conditions (30 lux). An experimental SD rat was placed inside the arena, and its movements were monitored using an infrared camera for two consecutive sessions of $2.5 \mathrm{~min}$ each. During the first session ("No target"), an empty wire mesh cage $(30 \times 15 \times 15 \mathrm{~cm})$ was placed at one end of the field. During the second session ("target"), an unfamiliar $\mathrm{BN}$ or $\mathrm{SD}$ rat was placed in the mesh cage. Time spent in the interaction zone was quantified using custom applications (Time OFCR4, O'Hara \& Co., Tokyo, Japan). The interaction ratio was calculated as (interaction time, "target")/(interaction time, "No target") $\times 100 \%$.

\section{Fecal and blood sample collection}

Fresh fecal samples were collected before the first SDS application (before), 1 day (stress 2d), 4 days (stress $5 \mathrm{~d}$ ), and 10 days (stress 11d) after the first SDS application, and 1 day (after stress), 7 days $(1 \mathrm{~W})$, and 1 month $(1 \mathrm{M})$ after the last SDS, and stored at $-80^{\circ} \mathrm{C}$ until further analysis. After the end of SDS periods, blood plasma samples were collected via cardiac puncture under pentobarbital sodium (Somnopentyl; Kyoritsu, Tokyo, Japan) anesthesia $(60 \mathrm{mg} / \mathrm{kg}$, intramuscular $)$ for subsequent metabolite analysis.

\section{S rRNA gene sequencing analysis}

Bacterial genomic DNA in fecal samples was extracted, and then two-step polymerase chain reactions (PCRs) were performed. The first PCR utilized either universal primer pair corresponding to the $\mathrm{V} 3-\mathrm{V} 4$ region of the $16 \mathrm{~S}$ rRNA gene: (1) 341F (5'-CCTACGGGNGGCWGCAG$\left.3^{\prime}\right)$ and $805 \mathrm{R}$ (5'-GACTACHVGGGTATCTAATCC-3') or (2) 341F (5'-TCGTCGGCAGCGTCAGATGTGTA TAAGAGACAGCCTACGGGNGGCWGCAG-3 ${ }^{\prime}$ ) and 806R (5'-GTCTCGTGGGCTCGGAGATGTGTATAAG AGACAGGGACTACHVGGGTWTCTAAT-3') (TaKaRa Bio, Shiga, Japan). The second PCR was performed to add the index sequences for Illumina sequencing with barcode sequences. The prepared libraries were subjected to either paired-end 300 [for (1)] or 250 [for (2)] base sequencing, using the MiSeq Reagent Kit v3 on the MiSeq (Illumina, San Diego, CA, USA).

Microbiome analysis was performed using the microbial genomics module either on the CLC Genomics Workbench version 8.5.1 (Qiagen, Hilden, Germany) [for (1)] or the open-source bioinformatics pipeline Quantitative 
Insights Into Microbial Ecology (QIIME) version 1.8.0 [for (2)]. Sequenced paired-end reads were assembled to construct contigs; chimeric contigs were removed by applying either the chimera crossover detection algorithm [for (1)] or CD-HIT-operational taxonomic unit (OTU) algorithm [for (2)]. The remaining contigs were clustered into OTUs with $97 \%$ sequence similarity. To acquire taxonomic information for each OTU, representative sequences were assigned to the Greengenes $16 \mathrm{~S}$ rRNA database by RDP classifier (version 2.2).

\section{Analysis of sulfur metabolomics}

Sulfur metabolomics (Sulfur index) was performed using S-bimanyl derivatives via LC-MS/MS (Shimadzu Nexera UHPLC system with on-line LCMS 8040, Kyoto, Japan) as described previously ${ }^{13-16}$. Briefly, the sulfur-containing compounds were extracted from samples by adding methanol and converted to fluorescent derivatives using a thiol-specific alkylating reagent (monobromobimane). The target metabolite levels were determined from the peak area by mass chromatography and represented as relative amounts following normalization using the internal standard (D-camphor-10-sulfonic acid) peak area.

\section{In vitro study}

L. reuteri (JCM Nos. 1112, 5867,1081, 1084, 5868, 5869, RIKEN BRC, Japan) and Escherichia coli (DH5 $\alpha$, BW2) were cultured in GAM medium at $30^{\circ} \mathrm{C}$ for $24 \mathrm{~h}$. Bacteria and supernatants were then collected for Sulfur index analysis.

\section{Oral ingestion of L-ergothioneine}

Oral administration of L-ergothioneine $(0.25 \mathrm{mg} / \mathrm{ml}$; Nagase \& Co., Tokyo, Japan) aqueous solution was conducted from 1 week prior to SDS initiation (day -7) to the end SDS application (day 14). The concentration of Lergothioneine administered corresponds to $\sim 30 \mathrm{mg} / \mathrm{kg} /$ day based on the initial water intake and body weight. Tang et al. demonstrated that daily oral administration of ergothioneine (35 or $70 \mathrm{mg} / \mathrm{kg} /$ day for 1,7 , and 28 days) induces accumulation of ergothioneine in whole-body tissues, including the brain, in male C57BL6J mice ${ }^{20}$. Control animals received only water.

\section{Surgery and electroencephalogram (EEG) recording}

Under pentobarbital sodium anesthesia $(60 \mathrm{mg} / \mathrm{kg}$, intramuscular), the rats were fixed to a stereotaxic apparatus (SR-6M; Narishige, Tokyo, Japan). Non-polarized $\mathrm{Ag} / \mathrm{AgCl}$ screw electrodes (1 mm diameter, O'Hara \& Co., Tokyo, Japan) were implanted epidurally on the left side of the parietal cortex (LPa; $\mathrm{Br}-2.0, \mathrm{~L} 1.5)$. Reference and ground electrodes were placed above the cerebellum. An electromyography (EMG) stainless electrode surface was subcutaneously placed on the dorsal neck muscle. The lead wires from all electrodes were soldered to a small socket and mounted on the skull with acrylic resin cement, along with these electrodes. A recovery period ( $>7$ days) was scheduled before initiation of the experiment.

To collect the EEG data, the rat was moved to an experimental cage with a soundproof box in which the $12 \mathrm{~h}$ light/dark cycle was maintained and connected via a recording cable with a built-in operational amplifier (TL074; Texas Instruments, Dallas, TX, USA) to reduce electrical and movement artifacts. This stage was recorded for $27 \mathrm{~h}$ including the first $3 \mathrm{~h}$ of habituation and $24 \mathrm{~h}$ of recording, from 13:30 to 16:30. Signals from the LPa and EMG electrode, referenced to the cerebellum electrodes, were amplified and filtered (2000 and 5000 gain, 0.1 and $0.003 \mathrm{~s}$ time constant for EEG and EMG, respectively; high cut-off filter for both; AB-621G, NihonKoden, Tokyo, Japan) and digitized at a $500 \mathrm{~Hz}$ sampling rate (Power 1401; Cambridge Electronic Design, Cambridge, UK).

\section{Sleep scoring}

Sleep/wake stages were scored using the automatic scoring tool "rat sleep auto" script in Spike2 (Cambridge Electronic Design). Scores were calculated by analyzing the EEG and EMG signals in consecutive $30 \mathrm{~s}$ epochs. We also manually confirmed the scoring procedure accuracy using EEG derivatives and EMG signals to segregate the WAKE (desynchronized EEG and medium-high amplitude EMG), NREM (high-voltage slow-wave EEG and low-amplitude EMG), and REM (EEG theta activity and very low amplitude EMG) stages for each epoch during the $24 \mathrm{~h}$ recording period ${ }^{3}$.

\section{Statistical analyses}

Statistical analyses were mostly performed using GraphPad Prism 8 (La Jolla, CA, USA). For statistical comparisons of two groups, the Mann-Whitney $U$ test, as the nonparametric version of the parametric $t$-test, was used. For comparisons of more than two groups, analysis of variance (ANOVA) was used followed by post hoc tests. Alpha diversity was calculated using three different parameters: Chao1 richness estimator and Shannon and inverse Simpson indexes. Principal coordinate analysis was performed using XLSTAT software package (Addinsoft, Long Island City, NY, USA). All data represent the mean \pm SEM.

\section{Results}

\section{Gut microbiota analysis}

We studied fecal gut microbiota before (before), during (stress $5 \mathrm{~d}, 11 \mathrm{~d}$ ), and after (after stress, $1 \mathrm{~W}, 1 \mathrm{M}$ ) the 14 day SDS. SDS significantly increased the levels of fecal classes Betaproteobacteria and Flavobacteriia, while decreasing those of Clostridia (Fig. 1a, after stress). 


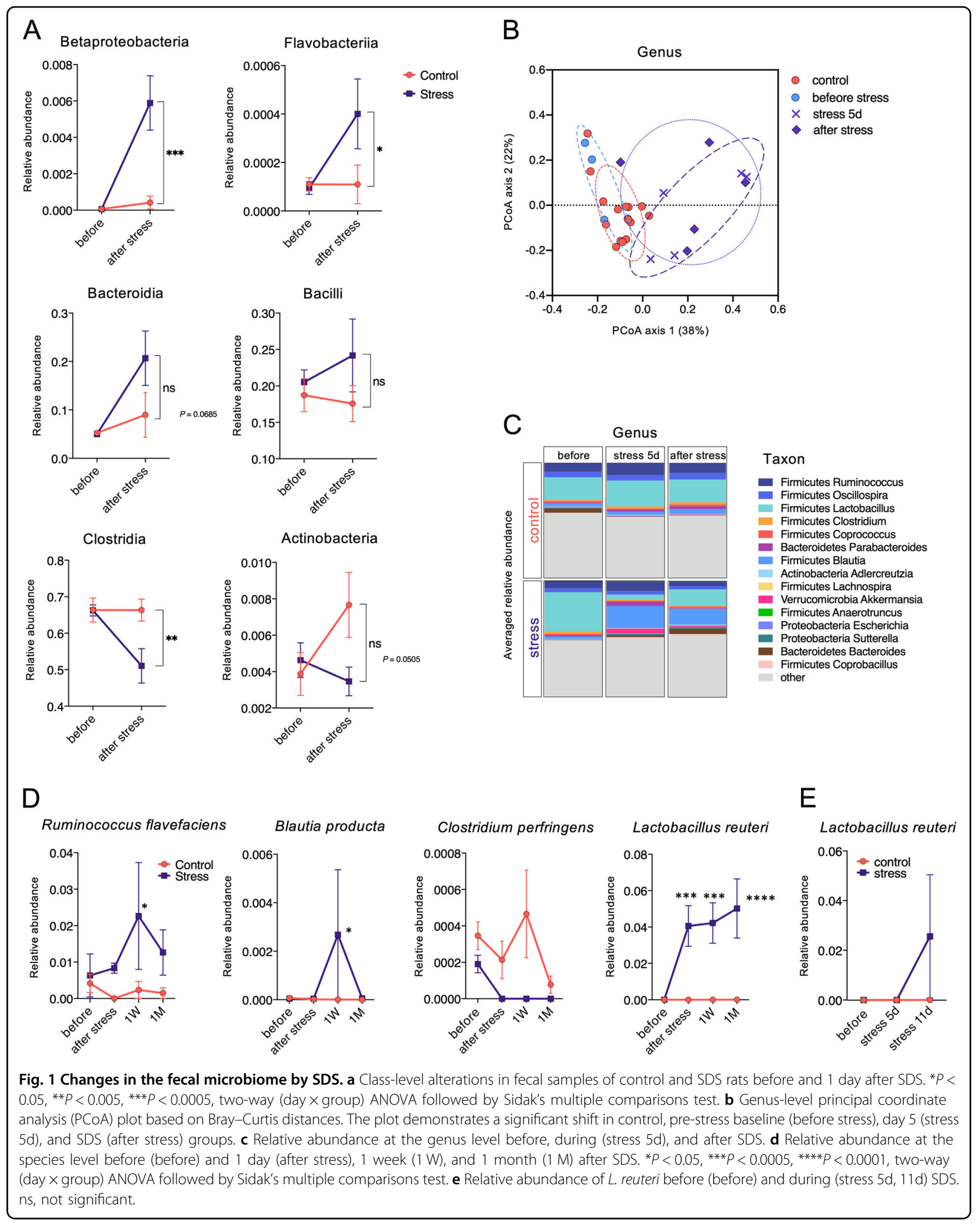


Bacteroidia and Bacilli showed a tendency to increase, whereas Actinobacteria tended to decrease.

Alpha diversity was analyzed to assess differences in within-subject diversity. The Chao1 richness estimator and Shannon and inverse Simpson indexes showed no statistically significant changes during and after SDS (Supplementary Fig. 1A-C). Analysis of beta diversity at the genus level was conducted to determine how the whole microbial community changed chronologically. Beta diversity exhibited no significant difference as assessed by Bray-Curtis distances before SDS (Fig. 1b; before). Bray-Curtis distances increased in the SDS rats at day 5 (Fig. 1b; stress $5 \mathrm{~d}$ ). Following SDS completion (Fig. 1b; after stress), Bray-Curtis distances further increased significantly $(P<0.0001)$.

Genus-level microbiota structure also markedly changed during the SDS period (Fig. 1c). When compared to before stress (before), Lactobacillus showed clear decreases at day 5, whereas Blautia exhibited large increases. These trends were fairly consistent after the last SDS exposure.

At the species level, $L$. reuteri levels significantly increased following SDS (Fig. 1d), with further increases observed even one month after SDS completion (Fig. 1d). Conversely, other species (Ruminococcus flavefaciens, Blautia producta, and Clostridium perfringens) exhibited only temporary change (Fig. 1d). More precise chronological analysis during the SDS period further revealed an increase in L. reuteri levels later in the SDS period (stress 11d) (Fig. 1e).

\section{Sulfur metabolite analyses}

Next, we analyzed SDS-induced changes in the fecal sulfur metabolites. The Sulfur index before and after SDS revealed significant decreases of glucose, methionine, and serine levels in the SDS rats (Fig. 2a). However, only ergothioneine, a natural thiol compound present in mushrooms and mammalian tissues ${ }^{21,22}$, exhibited a significant increase following SDS. We then evaluated chronological changes in the fecal ergothioneine levels in the SDS rats. A substantial increase in the amount of fecal ergothioneine was observed at day 11 (Fig. 2b). Statistically significant increases in the fecal ergothioneine levels were maintained even one month after the last SDS (Fig. 2b).

We also evaluated whether the fecal ergothioneine levels coordinated with plasma levels. Plasma ergothioneine levels one day following SDS did not significantly differ between control and SDS rats (Fig. 2c). Furthermore, plasma and fecal ergothioneine levels did not significantly correlate (Fig. 2d), indicating that although SDS increases fecal ergothioneine levels, ergothioneine may not be absorbed by the large intestinal tract, or the fecal levels may be insufficient to increase plasma levels.

\section{Production of ergothioneine by L. reuteri}

As ergothioneine biosynthesis occurs only in fungi or mycobacteria but not in mammals ${ }^{23,24}$, and chronological changes in L. reuteri and ergothioneine fecal levels were comparable in SDS rats, we hypothesized that the increase in the fecal ergothioneine levels in the SDS rats may be related to changes in gut microbiota. We, therefore, comprehensively examined the correlation between all gut bacterial species and all evaluated sulfur metabolites one day following SDS. Among gut bacteria, L. reuteri levels most significantly correlated with ergothioneine (Fig. 3a; $P<0.0001, r=0.82$ ). Moreover, the relative abundance of $L$. reuteri significantly positively correlated with fecal ergothioneine amounts when all chronological samples were analyzed together (Fig. 3b; $P<0.0001, r=0.8310$ ).

To directly demonstrate that $L$. reuteri produces ergothioneine, we conducted $L$. reuteri and $E$. coli culture experiments. Ergothioneine levels in the bodies and supernatants of $L$. reuteri were much higher than those of E. coli (Fig. 3c).

\section{Effects of L-ergothioneine administration on the SDS- induced depressive behavior and sleep abnormalities}

Ergothioneine has been shown to accumulate at high concentrations ( $100 \mu \mathrm{M}$ to $2 \mathrm{mM})$ in most cells and tissues of mammals including the brain ${ }^{25-27}$. Carnitine/ organic cation transporter 1 (OCTN1) ${ }^{28}$, encoded by the gene $S L C 22 A 4$, plays a pivotal role in this intracellular accumulation. Indeed, silencing this gene completely inhibits uptake of ergothioneine ${ }^{22,27}$. As the ileum expresses OCTN1 most abundantly in the body ${ }^{28,29}$, dietary ergothioneine is considered to be mainly absorbed in the ileum ${ }^{22,27}$ and is subsequently transported across the blood-brain barrier into the brain ${ }^{30}$. We, therefore, evaluated whether the protective properties of ergothioneine extend to the whole organism upon oral administration in SDS rats. We orally administered $0.25 \mathrm{mg} / \mathrm{ml} \mathrm{L-}$ ergothioneine as a preventative measure 7 days prior to SDS (day -7) and continued oral ingestion until the end of the SDS period (Fig. 4a). Interaction tests immediately following SDS showed that SDS rats exhibited significant social avoidance behaviors against unfamiliar BN or SD rats (Fig. 4b). Preventative L-ergothioneine administration resulted in statistically significant improvements in the avoidance behaviors against resident $\mathrm{BN}$ rats $(P=0.0198)$ and almost significant improvements against $\mathrm{SD}$ rats $(P=$ 0.0542) immediately after SDS (Fig. 4b; after stress). These significant improvements in avoidance behaviors against $\mathrm{BN}$ rats were still observed one month after the last SDS $(P=0.0130$, Fig. $4 \mathrm{~b} ; 1 \mathrm{M})$.

SDS also induced significant abnormalities in sleep such as increased REM sleep duration (Fig. 4c, d), shortened REM latency (Fig. 4e), and increased numbers of fragmentations (Fig. 4f) during the light phase. Lergothioneine ingestion significantly improved these REM sleep abnormalities except for the REM sleep latency. Moreover, in NREM sleep, SDS significantly 


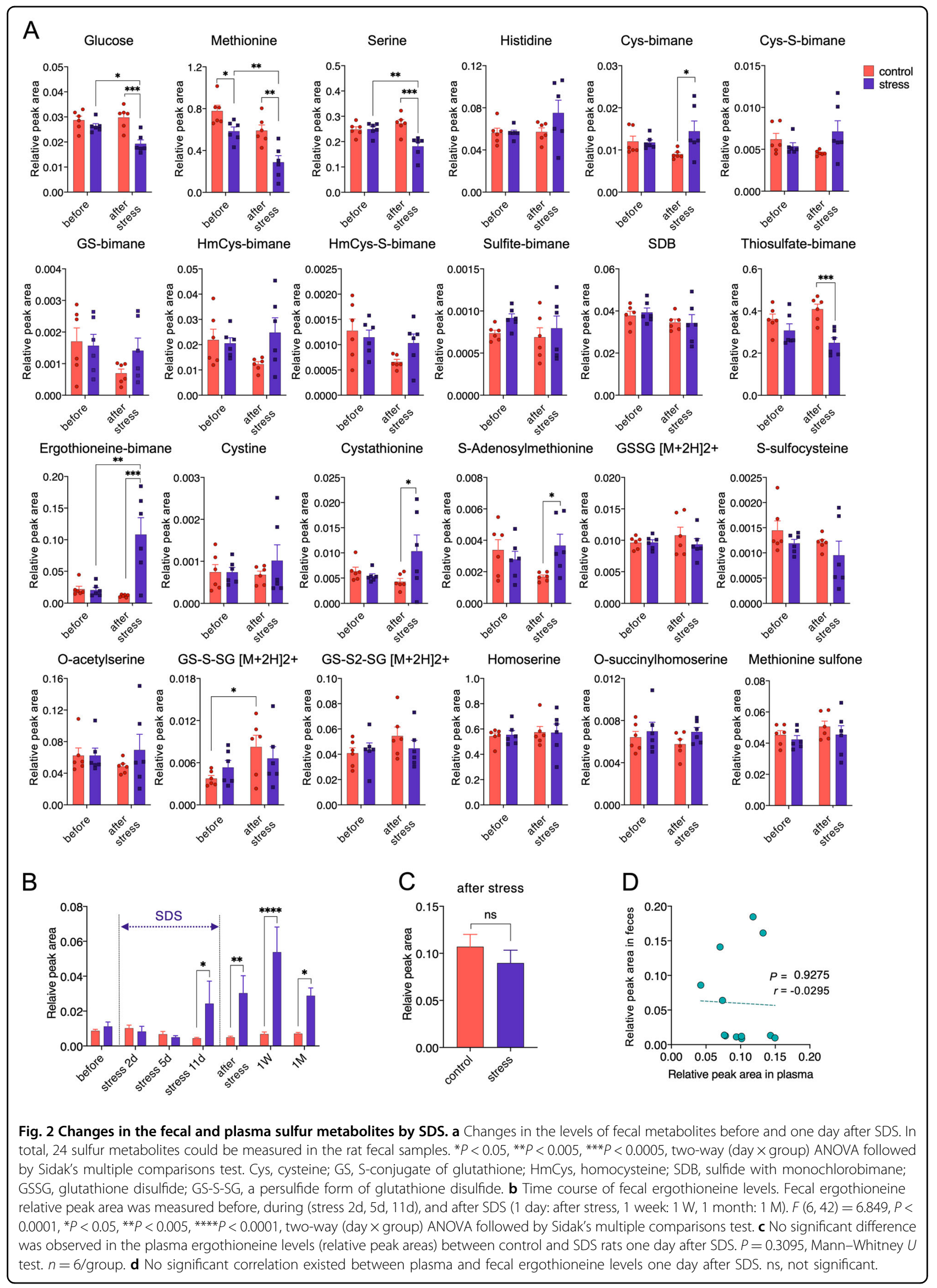



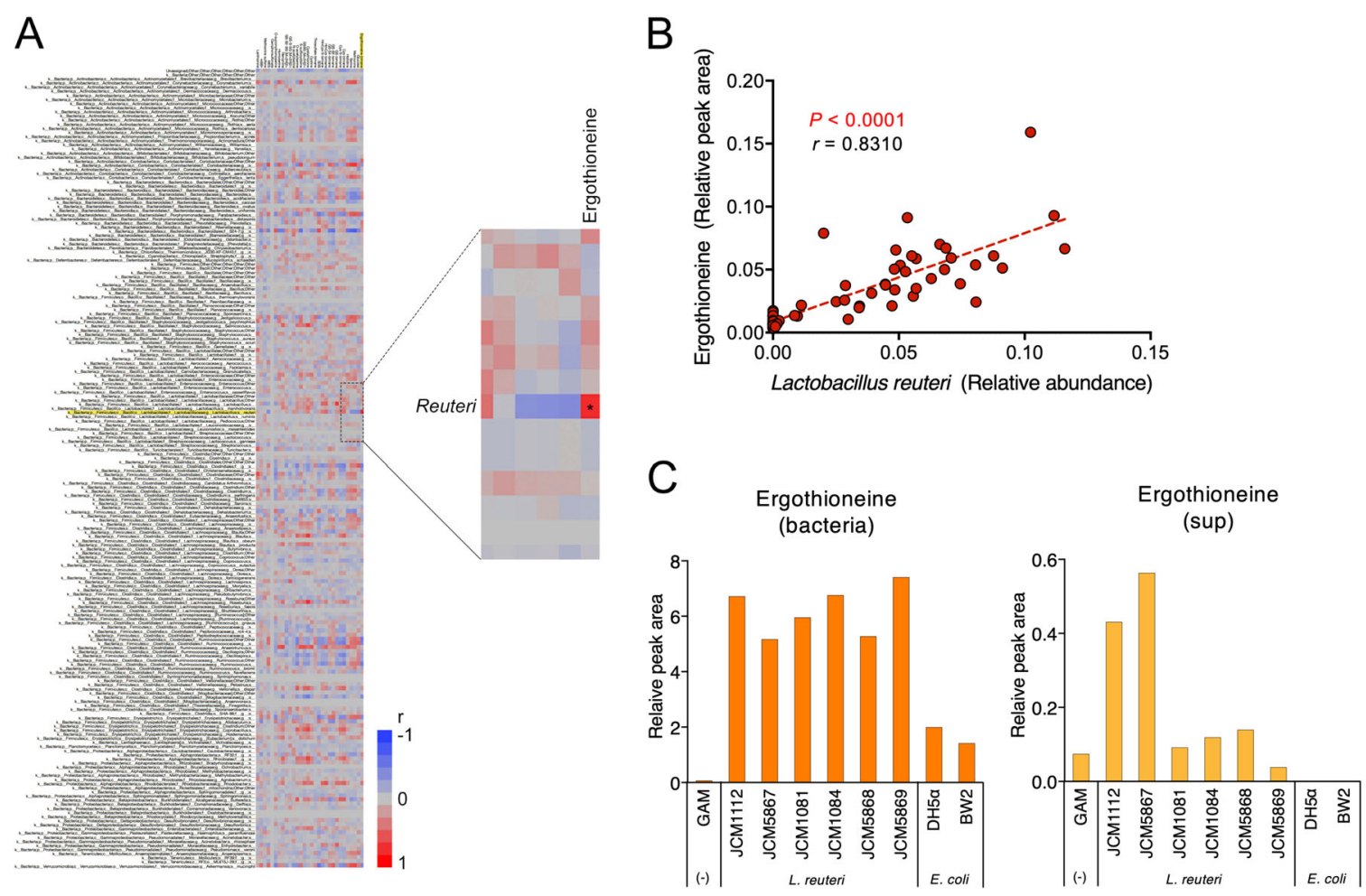

Fig. 3 Ergothioneine production by L. reuteri. a Correlation analysis of fecal gut bacterial (species level) and sulfur metabolites. The enlarged image shows a strong positive correlation in the levels between the gut bacterium $L$. reuteri and the sulfur metabolite ergothioneine. b Significant positive correlation between fecal $L$. reuteri bacterial relative areas and ergothioneine amounts. $P<0.0001, r=0.8310$. c Ability of $L$. reuteri to produce ergothioneine in vitro. Relative peak areas of ergothioneine in the bacterial bodies (left; bacteria) and supernatants (right; sup) of $L$. reuteri or E. coli are shown.

decreased the duration (Fig. 4d) and increased the fragmentation number (Fig. 4f), whereas L-ergothioneine tended to improve these abnormalities.

Finally, microbial diversity was analyzed in the feces from control, stressed, and L-ergothioneine-treated rats. Alpha diversity in the feces of SDS rats tended to be lower $(P=$ 0.0582) based on the Chaol estimator when compared to that of controls, but other indexes (Shannon and inverse Simpson) showed no statistically significant difference among the three groups (Supplementary Fig. 1D-F).

Beta diversity analysis at the genus level revealed that L-ergothioneine administration has a preventative effect on the abnormal shift of the whole microbial community induced by SDS (Fig. 4g). L-ergothioneine administration significantly increased the levels of fecal ergothioneine (approximately five times) (Fig. 4h), suggesting that biologically relevant amounts of ergothioneine were most likely absorbed from the ileum via OCTN1 in our paradigm, and the remainder was excreted in the feces.

\section{Discussion}

The major findings in this study were as follows. (1) Increases in the amount of fecal $L$. reuteri were observed 11 days after the initiation of SDS. The levels of $L$. reuteri continued to increase significantly and were retained at least for one month following the last SDS. (2) The Sulfur index showed significant increases in the levels of fecal ergothioneine 11 days after SDS initiation. These increases peaked at one week and remained significantly increased one month after the last SDS. (3) The increased amounts of fecal $L$. reuteri significantly positively correlated with the ergothioneine levels. We further found that L. reuteri directly produced ergothioneine in vitro. (4) Given the anti-inflammatory and anti-oxidative actions of ergothioneine, we orally administered L-ergothioneine to the SDS rats as a preventative measure and found that the social avoidance behaviors were ameliorated. We additionally observed improvements in sleep abnormalities, particularly those relating to REM sleep.

Previous studies showed that the feces transplanted from patients with MDD caused MDD-like behavior in germ-free animals ${ }^{10,11}$, suggesting that the proportion of harmful "depression bacteria" may predominate in the feces of these patients ${ }^{11}$. At the genus level, Coprococcus and Dialister, so-called "depression bacteria", are known to increase in the feces of patients with MDD and induce 
A

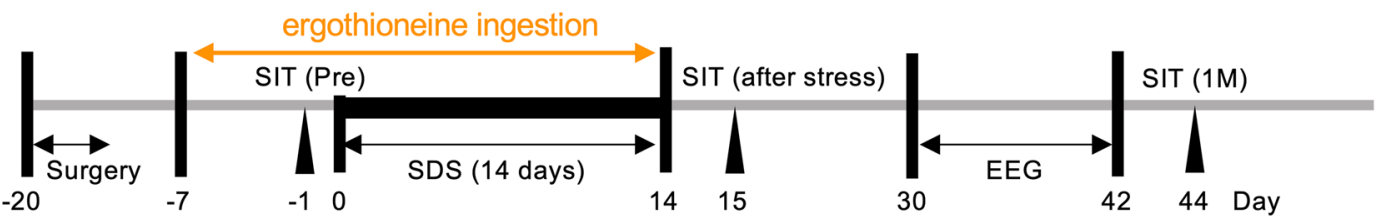

B
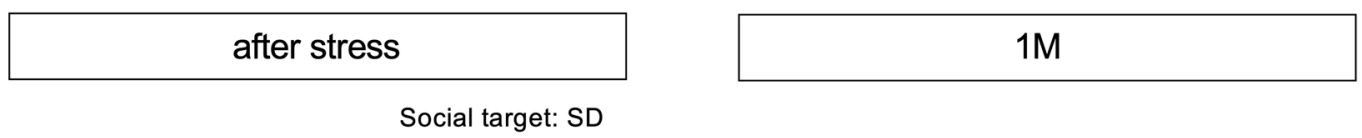

Social target: BN

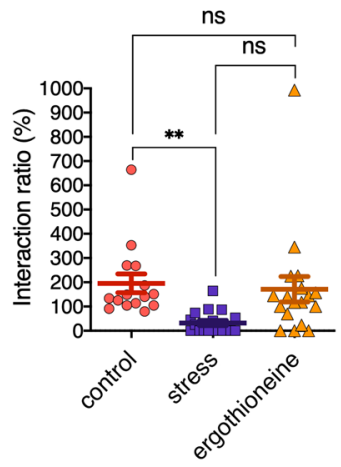

Social target: BN

Social target: SD
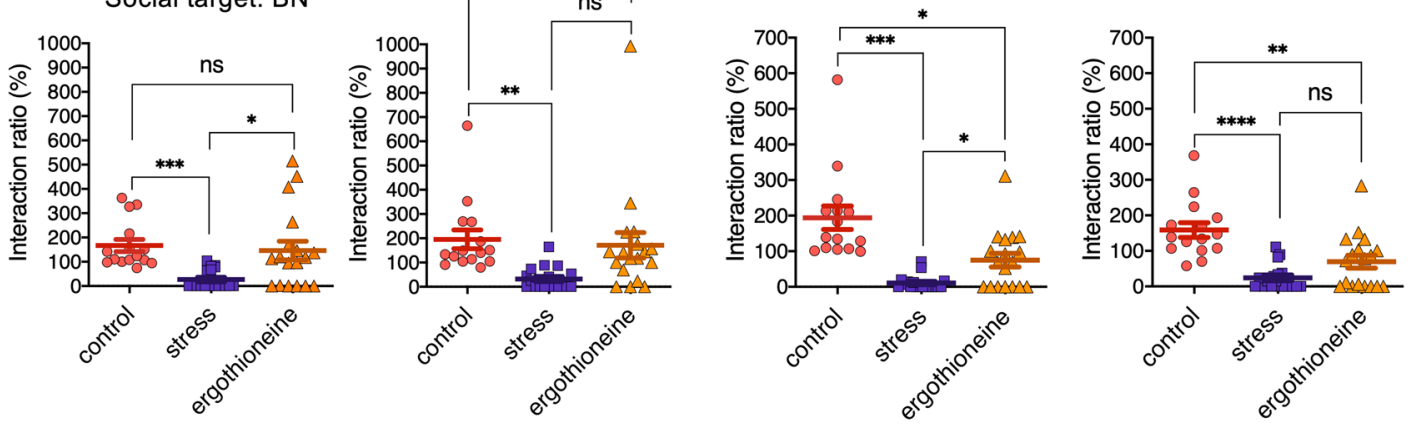

C
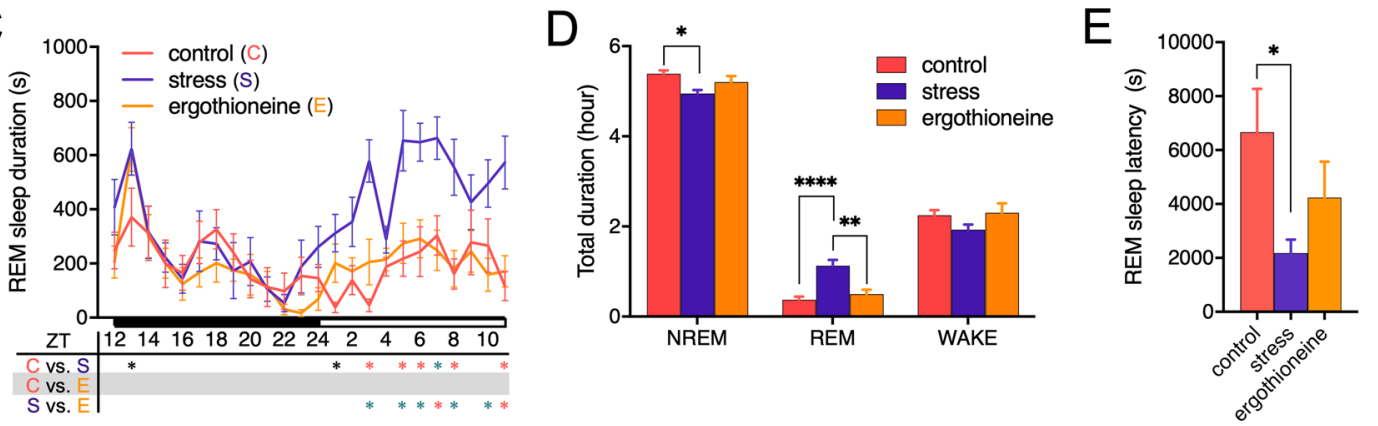

F
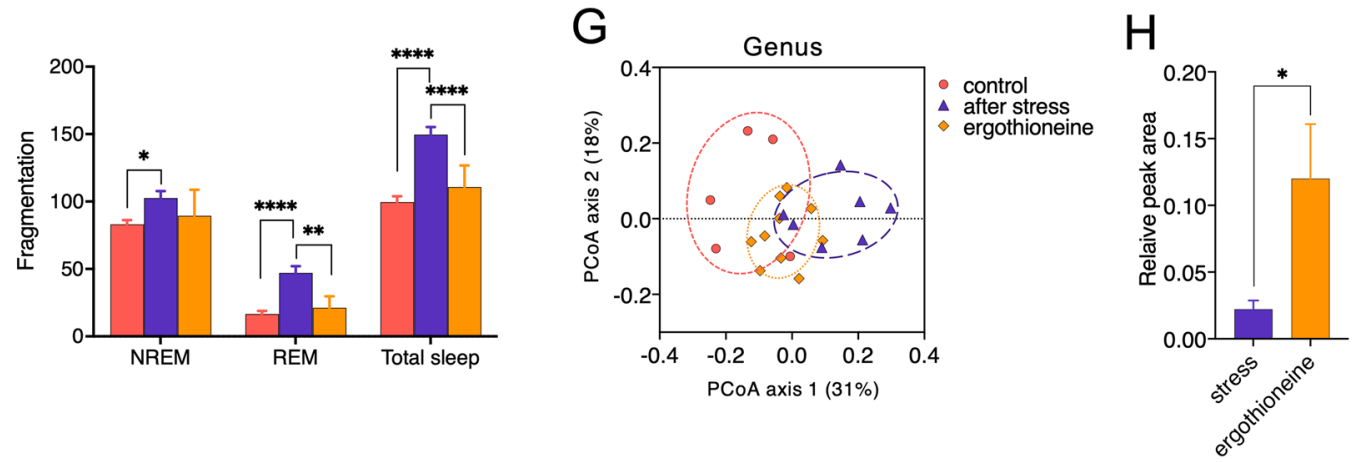

Fig. 4 Antidepressant effects of oral L-ergothioneine ingestion in the SDS rat model. a Experimental design of oral L-ergothioneine ingestion, SDS session, social interaction test, and $24 \mathrm{~h} \mathrm{EEG} \mathrm{recording.} \mathrm{b} \mathrm{Social} \mathrm{interaction} \mathrm{test} \mathrm{one} \mathrm{day} \mathrm{or} \mathrm{one} \mathrm{month} \mathrm{following} \mathrm{SDS} \mathrm{(day} 15$ or day 44 in (a), $n=$ 15-18/group). ${ }^{*} P<0.05,{ }^{* *} P<0.005,{ }^{* *} P<0.0005,{ }^{* * * *} P<0.0001$, one-way ANOVA (Welch and Browne-Forsythe tests) followed by Dunnett's T3 multiple comparisons test. c REM sleep variation. $F(46,506)=2.260, P<0.0001,{ }^{*} P<0.05$ (black), ${ }^{*} P<0.005$ (green), ${ }^{*} P<0.001$ (red), two-way (time $\times$ group) repeated ANOVA followed by Tukey's multiple comparisons test. ZT, Zeitgeber time. $n=7-10 /$ group. d Total duration in NREM, REM sleep, and wakefulness (WAKE) during the light phase [08:00 (ZTO) - 16:30 (ZT8.5)]. $F(4,66)=9.613, P<0.0001,{ }^{*} P<0.05,{ }^{* *} P<0.005,{ }^{* * * *} P<0.0001$, twoway (sleep stage $\times$ group) ANOVA followed by Tukey's test. $n=7-10 /$ group. e REM sleep latency. $F(2,22)=4.033, P=0.0322,{ }^{*} P<0.05$, one-way ANOVA followed by Tukey's test. $\mathbf{f}$ Total number of fragmentations of NREM, REM sleep, and WAKE during the light phase $(Z T O-Z T 8.5)$. $F(4,66)=$ $3.000, P=0.0245,{ }^{*} P<0.05,{ }^{* *} P<0.005,{ }^{* * *} P<0.0001$, two-way (sleep stage $\times$ group) ANOVA followed by Tukey's test. $n=7-10 / \mathrm{group}$. $\mathbf{g}$ Recovery of the abnormal shift in the SDS rat microbiota by L-ergothioneine. $\mathbf{H}$ Fecal ergothioneine levels following L-ergothioneine oral administration. $P=$ 0.0185, Mann-Whitney U test. 
MDD-like behaviors in animals ${ }^{31}$; however, we found no such changes related to these bacteria in our study (Fig. 1c). Rather, we found a quite unique long-lasting increase in the levels of $L$. reuteri in our SDS rat model (Fig. 1d, e). L. reuteri, a potential probiotic known to modulate the immune system ${ }^{32}$, may decrease anxiety as measured on the elevated plus maze ${ }^{33}$, reduce the stressinduced increase of corticosterone levels ${ }^{34}$, and reduce despair-like behavior in mice ${ }^{35,36}$. A similar increase in the level of $L$. reuteri was noted in a rat SDS model with a 7 day SDS regimen ${ }^{13}$. Resilient rats showed a greater increase in $L$. reuteri levels than did vulnerable rats; however, further mechanistic studies were not performed ${ }^{13}$.

In the present study, we also found a significant sharp increase in the levels of the sulfur metabolite ergothioneine in the feces of SDS rats in the second half of the SDS period, with this characteristic increase also continuing for at least a month following the cessation of stresses. The biosynthesis of ergothioneine, a highly stable anti-oxidative and anti-inflammatory sulfur metabolite $^{23,37-40}$, occurs only in fungi or mycobacteria ${ }^{23,24}$, suggesting that the changes in the gut microbiota may cause such increase. As expected, comprehensive correlation analysis demonstrated that fecal ergothioneine and $L$. reuteri levels significantly correlated. In addition, in vitro study further demonstrated that $L$. reuteri exhibits considerably greater ergothioneine production capability than does E. coli. Given the well-documented antiinflammatory effects of Lactobacillus ${ }^{32}$ and ergothioneine $e^{23,37-40}$, these findings suggest that an antiinflammatory defense mechanism mediated via $L$. reuteri and its sulfur metabolite ergothioneine may occur in the lower gastrointestinal tract as a result of long-term psychosocial stress. This concept was further supported by preliminary experiments involving fecal S100A9 (Supplementary Fig. 2), a protease-resistant neutrophil-derived protein $^{41-43}$ that can be used to predict relapse of inflammatory bowel diseases ${ }^{43-45}$. We found that the levels or rate of increase of S100A9 was elevated in the feces of SDS rats but returned to control levels by oral ergothioneine administration (Supplementary Fig. 2), suggesting the occurrence of bowel inflammation due to SDS and anti-inflammatory effects of ergothioneine. In our rat model, however, this defense mechanism may not be efficiently reflected in the body as no difference was observed in the plasma ergothioneine levels.

In addition to its anti-oxidative actions, ergothioneine also exerts several cytoprotective functions such as lipid peroxidation inhibition ${ }^{46,47}$ and DNA/protein damage control $^{23,48}$. A study using control mice reported the antidepressant-like effects of ergothioneine in forced swim and tail suspension tests ${ }^{30}$; however, to our knowledge, no study has examined the effect of ergothioneine in stressed animal models. We, therefore, investigated the effects of oral ergothioneine administration on MDD-like behavior and sleep abnormalities in our SDS rat model and confirmed its preventative effects. Although the functional mechanism associated with the antidepressant effect of ergothioneine remains unknown, mounting evidence suggests that increased central or peripheral inflammatory processes may be involved in stress-related mood disorders, such as $\mathrm{MDD}^{30,49-52}$. Consistent with this, inflammatory cytokines are elevated in patients with depression ${ }^{53-56}$. Furthermore, numerous animal studies have shown that inflammatory mechanisms underlie stress-induced depressive-type behaviors ${ }^{53-56}$. Therefore, it is plausible that, following absorption, ergothioneine may manifest its antidepressant effects through antiinflammatory mechanisms of the central and/or peripheral nervous system.

Several possibilities underlying ergothioneine-mediated rescue of sleep abnormalities should be mentioned. First, neuronal circuit-level dysregulation of the sleep stage transition in the brainstem region ${ }^{57-59}$ occurred consequent to SDS-associated inflammation, which may be prevented by the anti-inflammatory effects of ergothioneine as described above. Second, abnormalities in endogenous sleep substances (e.g., prostaglandin D2 or adenosine), including reduced glutathione, which plays an important role in removing reactive oxygen species, may also be involved in the sleep abnormalities ${ }^{60,61}$. For example, reduced amounts of glutathione were observed in the brains of acutely sleep-deprived rats ${ }^{62,63}$. More recently, a study using short-sleeping Drosophila mutants revealed a bidirectional relationship between oxidative stress and sleep: oxidative stress triggers sleep, which then acts as an antioxidant for both the body and the brain ${ }^{64}$. In this context, the concept of treatment and prevention of depression-related sleep abnormalities by controlling intracerebral oxidative stress production may be groundbreaking, as ergothioneine may harbor enormous potential as a direct sleep improvement tool.

Limitations of this study should be noted. First, the mechanisms underlying improvement of the MDD-like symptoms in the SDS rats by oral ergothioneine administration remain unelucidated. It is plausible that oral ergothioneine simply ameliorates SDS-induced inflammatory events in the periphery (especially in the intestinal flora), which in turn inhibits the MDD-like symptoms via unknown mechanisms. Second, we did not produce any CNS data related to disturbed sleep and inflammation. This is partly because of the lack of knowledge regarding the CNS center responsible for disturbed sleep associated with MDD, a major behavioral phenotype recapitulated in the current model. Nonetheless, we focused on the septohippocampal pathways that generate the hippocampal REM theta oscillation ${ }^{65}$, since REM theta powers most significantly correlated with the MDD-like social 
interactions in our SDS rats treated with antidepressants ${ }^{3}$ and L-ergothioneine (Supplementary Fig. 3). Further study is warranted to clarify the related CNS changes. Finally, we cannot exclude the possibility that the bacterial flora of SDS-administered SD rats was influenced by that of $\mathrm{BN}$ rats, considering that they had direct contact.

In conclusion, for the first time, we demonstrated the role of $L$. reuteri and identified its sulfur metabolite ergothioneine as a candidate molecular inhibitor of psychosocial stress in the microbiota-gut-brain axis $^{4-9}$. Oral L-ergothioneine ingestion significantly prevented MDDlike social avoidance and sleep abnormalities in our SDS rats. Considering that ergothioneine is a natural ingredient that is incorporated into the blood via OCTN1 mainly in the ileum ${ }^{20}$, our data warrant future evaluation of preventative use of ergothioneine in stress-related diseases including MDD. Furthermore, increases in fecal ergothioneine may serve as an effective MDD biomarker, at least for a subtype.

\section{Acknowledgements}

The authors thank Ms. Moe Watanabe for her technical assistance. We would like to thank Norihito Murayama and Hiroshi Watanabe from the Suntory Global Innovation Center for their technical suggestions. This study was funded by JSPS KAKENHI Grant Numbers 25871162 and 16 K04442 to Y.M. and $15 \mathrm{~K} 15438$ to $\mathrm{Y} . \mathrm{T}$.

\section{Author details}

${ }^{1}$ Affective Disorders Research Project, Tokyo Metropolitan Institute of Medical Science, Tokyo, Japan. ${ }^{2}$ Research Institute, Suntory Global Innovation Center Ltd., Kyoto, Japan. ${ }^{3}$ Graduate School of Biological Sciences, Nara Institute of Science and Technology, Nara, Japan. ${ }^{4}$ Faculty of Life and Environmental Sciences, University of Tsukuba, Ibaraki, Japan

\section{Author contributions}

Y.M., K.W., K.S., and Y.T. designed the experiments; Y.M., N.O., T.S., K.W., Y.K., I.O., and Y.T. performed the experiments; Y.M., K.W., K.S., and Y.T. designed the analyses and discussed the results; Y.M., K.W., K.S., Y.K., I.O., and Y.T. analyzed the data; Y.M. and Y.T. wrote the paper; Y.M. and N.O. performed animal surgeries; and all authors commented on the paper.

\section{Conflict of interest}

This study was supported by the Foundation for Life Science of the Suntory Global Innovation Center. Y.T. received financial assistance for this study. Y.M., N.O., T.S., K.S., Y.K., and I.O. declare no conflict of interest.

\section{Publisher's note}

Springer Nature remains neutral with regard to jurisdictional claims in published maps and institutional affiliations.

Supplementary Information accompanies this paper at (https://doi.org/ 10.1038/s41398-020-0855-1).

Received: 28 December 2019 Revised: 22 April 2020 Accepted: 7 May 2020 Published online: 28 May 2020

\footnotetext{
References

1. Krishnan, V. \& Nestler, E. J. The molecular neurobiology of depression. Nature 455, 894-902 (2008).

2. Tsuno, N., Besset, A. \& Ritchie, K. Sleep and depression. J. Clin. Psychiatry 66, 1254-1269 (2005).
}

3. Matsuda, Y. et al. Chronic antidepressant treatments rescue reduced REM sleep theta power in a rat social defeat stress model of depression. Preprint at https://www.biorxiv.org/content/10.1101/2020.03.21.001164v1 (2020).

4. Sudo, N. et al. Postnatal microbial colonization programs the hypothalamicpituitary-adrenal system for stress response in mice. J. Physiol. 558, 263-275 (2004).

5. Mayer, E. A. Gut feelings: the emerging biology of gut-brain communication. Nat. Rev. Neurosci. 12, 453-466 (2011).

6. Bravo, J. A. et al. Ingestion of Lactobacillus strain regulates emotional behavior and central GABA receptor expression in a mouse via the vagus nerve. Proc. Natl Acad. Sci. USA 108, 16050-16055 (2011).

7. Foster, J. A. \& McVey Neufeld, K. A. Gut-brain axis: how the microbiome influences anxiety and depression. Trends Neurosci. 36, 305-312 (2013).

8. Rogers, G. B. et al. From gut dysbiosis to altered brain function and mental illness: mechanisms and pathways. Mol. Psychiatry 21, 738-748 (2016).

9. Sarkar, A. et al. Psychobiotics and the manipulation of bacteria-gut-brain signals. Trends Neurosci. 39, 763-781 (2016).

10. Kelly, J. R. et al. Transferring the blues: depression-associated gut microbiota induces neurobehavioural changes in the rat. J. Psychiatr. Res. 82, 109-118 (2016).

11. Zheng, P. et al. Gut microbiome remodeling induces depressive-like behaviors through a pathway mediated by the host's metabolism. Mol. Psychiatry 21, 786-796 (2016).

12. Burokas, A. et al. Targeting the microbiota-gut-brain axis: prebiotics have anxiolytic and antidepressant-like effects and reverse the impact of chronic stress in mice. Biol. Psychiatry 82, 472-487 (2017).

13. Pearson-Leary, J. et al. The gut microbiome regulates the increases in depressive-type behaviors and in inflammatory processes in the ventral hippocampus of stress vulnerable rats. Mol. Psychiatry 25, 1068-1079.

14. Kawano, Y. et al. Involvement of the yciW gene in L-cysteine and L-methionine metabolism in Escherichia coli. J. Biosci. Bioeng. 119, 310-313 (2015).

15. Tanaka, N., Kawano, Y., Satoh, Y., Dairi, T. \& Ohtsu, I. Gram-scale fermentative production of ergothioneine driven by overproduction of cysteine in Escherichia coli. Sci. Rep. 9, 1895 (2019).

16. Nakajima, T. et al. Effects of thiosulfate as a sulfur source on plant growth, metabolites accumulation and gene expression in arabidopsis and rice. Plant Cell Physiol. 60, 1683-1701 (2019).

17. Kawano, Y., Shiroyama, M., Kanazawa, K., Suzuki, Y. A. \& Ohtsu, I. Development of high-throughput quantitative analytical method for L-cysteine-containing dipeptides by LC-MS/MS toward its fermentative production. AMB Express $\mathbf{9}$, 91 (2019).

18. Berton, O. et al. Essential role of BDNF in the mesolimbic dopamine pathway in social defeat stress. Science 311, 864-868 (2006).

19. Krishnan, V. et al. Molecular adaptations underlying susceptibility and resistance to social defeat in brain reward regions. Cell 131, 391-404 (2007).

20. Tang, R. M. Y., Cheah, I. K., Yew, T. S. K. \& Halliwell, B. Distribution and accumulation of dietary ergothioneine and its metabolites in mouse tissues. Sci. Rep. 8, 1601 (2018).

21. Ey, J., Schömig, E. \& Taubert, D. Dietary sources and antioxidant effects of ergothioneine. J. Agric. Food Chem. 55, 6466-6474 (2007).

22. Sugiura, T. et al. Functional expression of carnitine/organic cation transporter OCTN1/SLC22A4 in mouse small intestine and liver. Drug Metab. Dispos. 38, 1665-1672 (2010).

23. Paul, B. D. \& Snyder, S. H. The unusual amino acid L-ergothioneine is a physiologic cytoprotectant. Cell Death Differ. 17, 1134-1140 (2010).

24. Fujitani, Y., Alamgir, K. M. \& Tani, A. Ergothioneine production using Methylobacterium species, yeast, and fungi. J. Biosci. Bioeng. 126, 715-722 (2018).

25. Mayumi, T. et al. Studies on ergothioneine. $V$. Determination by high performance liquid chromatography and application to metabolic research. Chem. Pharm. Bull. (Tokyo) 26, 3772-3778 (1978).

26. Kaneko, I. et al. Quantitative determination of ergothioneine in plasma and tissues by TLC-densitometry. Chem. Pharm. Bull. (Tokyo) 28, 3093-3097 (1980)

27. Kato, Y. et al. Gene knockout and metabolome analysis of carnitine/organic cation transporter OCTN1. Pharm. Res. 27, 832-840 (2010).

28. Gründemann, D. et al. Discovery of the ergothioneine transporter. Proc. Natl Acad. Sci. USA 102, 5256-5261 (2005).

29. Taubert, D., Jung, N., Goeser, T. \& Schömig, E. Increased ergothioneine tissue concentrations in carriers of the Crohn's disease risk-associated 503F variant of the organic cation transporter OCTN1. Gut 58, 312-314 (2009). 
30. Nakamichi, N. et al. Food-derived hydrophilic antioxidant ergothioneine is distributed to the brain and exerts antidepressant effect in mice. Brain Behav. 6, e00477 (2016).

31. Valles-Colomer, M. et al. The neuroactive potential of the human gut microbiota in quality of life and depression. Nat. Microbiol. 4, 623-632 (2019).

32. Liu, Y., Fatheree, N. Y., Mangalat, N. \& Rhoads, J. M. Human-derived probiotic Lactobacillus reuteri strains differentially reduce intestinal inflammation. Am. J. Physiol. Gastrointest. Liver Physiol. 299, G1087-G1096 (2010).

33. Sovijit, W. N. et al. Ovarian progesterone suppresses depression and anxiety-like behaviors by increasing the Lactobacillus population of gut microbiota in ovariectomized mice. Neurosci. Res. https://doi.org/10.1016/j. neures.2019.04.005 (2019)

34. Jang, H. M., Lee, K. E. \& Kim, D. H. The preventive and curative effects of Lactobacillus reuteri NK33 and Bifidobacterium adolescentis NK98 on immobilization stress-induced anxiety/depression and colitis in mice. Nutrients $\mathbf{1 1}$ E819 (2019).

35. Marin, I. A. et al. Microbiota alteration is associated with the development of stress-induced despair behavior. Sci. Rep. 7, 43859 (2017).

36. Waclawiková, B. \& El Aidy, S. Role of microbiota and tryptophan metabolites in the remote effect of intestinal inflammation on brain and depression. Pharm. (Basel) 11, E63 (2018).

37. Halliwell, B., Cheah, I. K. \& Tang, R. M. Y. Ergothioneine - a diet-derived antioxidant with therapeutic potential. FEBS Lett. 592, 3357-3366 (2018).

38. Cheah, I. K. \& Halliwell, B. Ergothioneine; antioxidant potential, physiological function and role in disease. Biochim. Biophys. Acta 1822, 784-793 (2012).

39. Cumming, B. M., Chinta, K. C., Reddy, V. P. \& Steyn, A. J. C. Role of ergothioneine in microbial physiology and pathogenesis. Antioxid. Redox Signal. 28, 431-444 (2018).

40. Song, T. Y. et al. Ergothioneine and melatonin attenuate oxidative stress and protect against learning and memory deficits in C57BL/6J mice treated with D-galactose. Free Radic. Res. 48, 1049-1060 (2014).

41. Dale, I., Brandtzaeg, P., Fagerhol, M. K. \& Scott, H. Distribution of a new myelomonocytic antigen (L1) in human peripheral blood leukocytes. Immunofluorescence and immunoperoxidase staining features in comparison with lysozyme and lactoferrin. Am. J. Clin. Pathol. 84, 24-34 (1985).

42. Tibble, J. et al. A simple method for assessing intestinal inflammation in Crohn's disease. Gut 47, 506-513 (2000).

43. Xiang, J. Y., Ouyang, Q., Li, G. D. \& Xiao, N. P. Clinical value of fecal calprotectin in deter- mining disease activity of ulcerative colitis. World J. Gastroenterol. 14, 53-57 (2008).

44. Tibble, J. A., Sigthorsson, G., Bridger, S., Fagerthol, M. K. \& Bjarnason, I. Surrogate markers of intestinal inflammation are predictive of relapse in patients with inflammatory bowel disease. Gastroenterology 119, 15-22 (2000).

45. Sekiya, S. et al. Enzyme-linked immunosorbent assay for S100A9 in the stool of rats with dextran sulfate sodium-induced colitis. J. Immunol. Methods 439, 44-49 (2016).

46. Bedirli, A. et al. Ergothioneine pretreatment protects the liver from ischemiareperfusion injury caused by increasing hepatic heat shock protein 70. J. Surg. Res. 122, 96-102 (2004).
47. Deiana, M. et al. L-ergothioneine modulates oxidative damage in the kidney and liver of rats in vivo: studies upon the profile of polyunsaturated fatty acids. Clin. Nutr. 23, 183-193 (2004).

48. Zhu, B. Z. et al. Ergothioneine prevents copper-induced oxidative damage to DNA and protein by forming a redox-inactive ergothioneine-copper complex. Chem. Res. Toxicol. 24, 30-34 (2011).

49. Felger, J. C., Haroon, E. \& Miller, A. H. Risk and resilience: animal models shed light on the pivotal role of inflammation in individual differences in stressinduced depression. Biol. Psychiatry 78, 7-9 (2015).

50. Piletz, J. E. et al. Pro-inflammatory biomakers in depression: treatment with venlafaxine. World J. Biol. Psychiatry 10, 313-323 (2009).

51. Wong, M. L. et al. Inflammasome signaling affects anxiety- and depressive-like behavior and gut microbiome composition. Mol. Psychiatry 21, 797-805 (2016).

52. Wood, S. K. et al. Inflammatory factors mediate vulnerability to a social stressinduced depressive-like phenotype in passive coping rats. Biol. Psychiatry 78, 38-48 (2015)

53. Felger, J. C. \& Lotrich, F. E. Inflammatory cytokines in depression: neurobiological mechanisms and therapeutic implications. Neuroscience 246, 199-229 (2013).

54. Raison, C. L. \& Miller, A. H. Is depression an inflammatory disorder? Curr. Psychiatry Rep. 13, 467-475 (2011).

55. Asnis, G. M. \& De La Garza, R. 2nd Interferon-induced depression: strategies in treatment. Prog. Neuropsychopharmacol. Biol. Psychiatry 29, 808-818 (2005).

56. Ma, L. et al. Animal inflammation-based models of depression and their application to drug discovery. Expert Opin. Drug Disco. 12, 995-1009 (2017).

57. Gottesmann, C. \& Gottesman, I. The neurobiological characteristics of rapid eye movement (REM) sleep are candidate endophenotypes of depression, schizophrenia, mental retardation and dementia. Prog. Neurobiol. 81, 237-250 (2007).

58. Hayashi, Y. et al. Cells of a common developmental origin regulate REM/nonREM sleep and wakefulness in mice. Science 350, 957-961 (2015).

59. Sapin, E. et al. Localization of the brainstem GABAergic neurons controlling paradoxical (REM) sleep. PLoS One 4, e4272 (2009).

60. Mizoguchi, A. et al. Dominant localization of prostaglandin D receptors on arachnoid trabecular cells in mouse basal forebrain and their involvement in the regulation of non-rapid eye movement sleep. Proc. Natl Acad. Sci. USA 98, 11674-11679 (2001).

61. Basheer, R., Strecker, R. E., Thakkar, M. M. \& McCarley, R. W. Adenosine and sleep-wake regulation. Prog. Neurobiol. 73, 379-396 (2004).

62. Ramanathan, L., Gulyani, S., Nienhuis, R. \& Siegel, J. M. Sleep deprivation decreases superoxide dismutase activity in rat hippocampus and brainstem. Neuroreport 13, 1387-1390 (2002).

63. D'Almeida, V. et al. Sleep deprivation induces brain region-specific decreases in glutathione levels. Neuroreport 9, 2853-2856 (1998).

64. Hill, V. M. et al. A bidirectional relationship between sleep and oxidative stress in Drosophila. PLoS Biol. 16, e2005206 (2018).

65. Boyce, R., Glasgow, S. D., Williams, S. \& Adamantidis, A. Causal evidence for the role of REM sleep theta rhythm in contextual memory consolidation. Science 352, 812-816 (2016). 